\title{
ESCORPIONISMO E INTELIGÊNCIA ARTIFICIAL: UMA INTERSECÇÃO PROMISSORA?
}

Jader Lúcio Pinheiro Sant'Ana, Oswaldo Jesus Rodrigues da Motta, Mathias Viana Vicari, Iram Borges de Moraes Rocha Filho, Gabriel Resende Machado, Eugênio da Silva, Luiz Alberto Santana. Escorpionismo e Inteligência Artificial: uma intersecção promissora? Revista Saúde Dinâmica, vol. 3, núm. 1, 2021. Faculdade Dinâmica do Vale do Piranga.

\section{SAÚDE DINÂMICA - Revista Científica Eletrônica FACULDADE DINÂMICA DO VALE DO PIRANGA}

7a Edição 2021 | Ano III - no 1 | ISSN - 2675-133X 


\title{
Escorpionismo e Inteligência Artificial: uma intersecção promissora?
}

\section{Scorpionism and Artificial Intelligence: a promising intersection?}

Jader Lúcio Pinheiro Sant'Ana ${ }^{1}$, Oswaldo Jesus Rodrigues da Motta ${ }^{2}$, Mathias Viana Vicari ${ }^{3}$, Iram Borges de Moraes Rocha Filho ${ }^{4}$, Gabriel Resende Machado ${ }^{5}$ Eugênio da Silva ${ }^{6}$, Luiz Alberto Santana ${ }^{7}$

${ }^{I}$ Curso de Medicina Veterinária, Faculdade de Ciências e Tecnologia de Viçosa (FAVIÇOSA/UNIVIÇOSA)

${ }^{2}$ Escola de Medicina, Faculdade Dinâmica do Vale do Piranga (FADIP); Departamento de Medicina e Enfermagem, Universidade Federal de Viçosa (DEM/UFV)

${ }^{3}$ Programa de Pós-graduação em Meteorologia Aplicada, Universidade Federal de Viçosa (UFV)

4; Escola de Medicina, Faculdade Dinâmica do Vale do Piranga (FADIP)

${ }^{5}$ Seção de Engenharia da Computação (SE/9) do Instituto Militar de Engenharia (IME)

${ }^{6}$ Curso de Ciência da Computação, Centro Universitário Serra dos Órgãos (UNIFESO); Unidade de Computação, Centro Universitário Estadual da Zona Oeste (UEZO)

${ }^{7}$ Departamento de Medicina e Enfermagem, Universidade Federal de Viçosa (UFV)

Autor correspondente: luizalbertosantana32@gmail.com

\section{Resumo}

Animais peçonhentos representam um grave problema de saúde pública, dado o elevado número de acidentes que causam. No Brasil, cinco espécies de escorpiões do gênero Tityus, são encontradas: Tityus serrulatus, Tityus bahiensis, Tityus stigmurus, Tityus paraensis e Tityus silvestris, sendo a espécie $T$. serrulatus, ou escorpião-amarelo, considerada a mais perigosa. O objetivo do estudo é apresentar revisão dos aspectos etiológicos, ecológicos, clínicos, epidemiológicos e profiláticos do escorpionismo e apresentar a Inteligência Artificial como método de estudo deste agravo à saúde. Os autores concluem que o entendimento sobre os sinais e os sintomas são essenciais para o diagnóstico rápido, elemento essencial para estabelecer o tratamento adequado, melhorando, assim, o prognóstico do paciente.

Palavras-chave: Animais Peçonhentos. Escorpiões. Escorpionismo. Inteligência Artificial.

\begin{abstract}
Venomous animals represent a serious public health problem, given the high number of accidents they cause. In Brazil, five species of scorpions of the genus Tityus, are found: Tityus serrulatus, Tityus bahiensis, Tityus stigmurus, Tityus paraensis and Tityus silvestris, being the species T. serrulatus, or yellow scorpion, considered the most dangerous. The objective of the study this study is to review the etiological, ecological, clinical, epidemiological and prophylactic aspects of scorpionism and to present Artificial Intelligence as a method of study of this health problem. The authors conclude that understanding of signs and symptoms is essential for rapid diagnosis, an essential element to establish adequate treatment, thus improving the patient's prognosis.

Key words: Venomous animals. Scorpionism. Artificial Intelligence.
\end{abstract}




\section{INTRODUÇÃO}

Escorpiões são artrópodes pertencente à classe Arachnida existentes há mais de 450 milhões de anos, sendo inicialmente pertencentes ao ambiente oceânico e estando entre os primeiros registros de aracnídeos que conquistaram o ambiente terrestre (POLIS 2001; BRASIL 2001). O empeçonhamento por estes animais é denominado escorpionismo e constitui um importante problema de saúde pública no Brasil, devido ao elevado número de acidentes anuais e com potencial de evolução para gravidade (TANIELE-SILVA, et al., 2020; LISBOA et al., 2020).

A gravidade do acidente, bem como os óbitos ocorridos, ocorre mais comumente nos extremos da vida. Entretanto, tais complicações também se associam a um diagnóstico tardio ou inadequado. Neste contexto, a Inteligência Artificial surge como instrumento de apoio diagnóstico para favorecer o reconhecimento da espécie envolvida no acidente e garantir o melhor prognóstico para a vítima (BRASIL, 2001; BRASIL, 2009; VELASCO et al., 2020; LOBO, 2018).

Os objetivos do presente estudo são (1) revisar os aspectos etiológicos, ecológicos e climáticos, clínicos, epidemiológicos e profiláticos dos acidentes por escorpião e (2) inovar a abordagem do problema a partir da discussão sobre o papel da Inteligência Artificial (IA) como ferramenta para a investigação destes eventos mórbidos.

\section{METODOLOGIA}

Para o desenvolvimento deste trabalho foram buscados documentos relacionados ao tema nas plataformas de dados científicos Scientific Eletronic Library Online (SciELO), Literatura Latino-Americana e do Caribe em Ciências da Saúde (LILACS), U. S. National Library of Medicine (PuBMed) e publicações de órgãos governamentais.

Durante a busca, foram utilizados os descritores "animais peçonhentos", "escorpiões", “escorpionismo" e "inteligência artificial”. Foram selecionados aqueles artigos que traziam dados relacionados aos diferentes aspectos clínicos e epidemiológicos do envenenamento por escorpiões em seres humanos, estudos do escorpionismo em território nacional e estudos sobre Inteligência Artificial que pudessem relacioná-la ao escorpionismo. Foram descartados trabalhos que não se relacionavam ao tema de interesse. Ao fim da seleção, todos os trabalhos passaram por leitura e 
análises críticas, sendo colhidos dados importantes para a confecção do manuscrito. Posteriormente, prosseguiu-se com a elaboração do texto final com as devidas considerações dos autores.

\section{BIOLOGIA DOS ESCORPIÕES E EPIDEMIOLOGIA DO ESCORPIONISMO}

Os escorpiões são artrópodes invertebrados de hábito noturno. Alimentam-se especialmente de outros pequenos artrópodes como grilos e baratas. Entretanto, podem ser presas de animais maiores como aranhas, lacraias, serpentes, lagartos, sapos e aves (SIQUEIRA-BATISTA et al., 2020). São animais pertencentes ao filo Arthropoda, à classe Arachnida, à ordem Scorpiones e à família Buthidae.

No Brasil, existe somente um único gênero de escorpiões; denominado Tityus. Cinco espécies são comumente encontradas no país, a saber: Tityus serrulatus, o escorpião-amarelo, Tityus bahiensis, o escorpião-marrom, Tityus stigmurus, o escorpião-do-nordeste, Tityus paraensis (também denominado Tityus obscurus), o escorpião preto da Amazônia e Tityus silvestris (BRASIL, 2001; BRASIL, 2009; BRASIL, 2019; COSTA et al., 2020).

O corpo desses animais é divido em cefalotórax e abdome. No cefalotórax se encontram as quelíceras, os pedipalpos - que são as clássicas pinças do animal - e quatro pares de pernas. No abdome, mais especificamente na parte ventral, encontramos o opérculo genital, apêndices sensoriais e os espiráculos, sendo estas últimas estruturas pertencentes ao sistema respiratório do artrópode. A cauda do escorpião se inicia na porção final do abdome e possui em sua extremidade uma estrutura denominada telson, na qual está situado o aguilhão - ou ferrão - do animal (BRASIL, 2009).

Os escorpiões são animais de hábito majoritariamente noturno e, durante o dia, podem ser encontrados refugiados sob troncos, entulhos, cascalhos de pedras, tijolos, telhas e madeiras acumuladas (BRASIL, 2009; BRASIL, 2019). Entretanto, com a crescente expansão das áreas urbanas em detrimento das áreas de reservas naturais, estes animais têm buscado abrigo nos espaços peridomiciliares e, por vezes, podem ser encontrados dentro de casas e habitações humanas, circunstância que justifica o aumento dos acidentes nos últimos anos (BRASIL, 2019).

Estima-se que, anualmente, ocorram entre 1,2 e 1,5 milhão de acidentes decorrentes do envenenamento por escorpiões em todo o mundo. Destes cerca de 2.600 acabam evoluindo para 
óbito (CHIPPAUX, 2012; CARMO et al., 2019). No Brasil, durante o período de 2013 a 2017 , ocorreram 895.608 acidentes envolvendo animais peçonhentos. Destes, o escorpionismo figurou como o acidente mais prevalente, contando, em 2013, com 78.363 casos e, em 2017, 124.077 casos, o que corresponde a uma elevação de 58,34\% no número de casos (SIQUEIRA- BATISTA et al., 2020). Em 2019, segundo o Sistema de Agravos de Notificação (SINAN), ocorreram 265.701 acidentes por animais peçonhentos no Brasil. Desta totalidade, 154.812 foram acidentes por escorpião, configurando 58,26\% do total de acidentes e 169 (0,1\% dos casos) evoluíram para óbito, sendo a maioria crianças. O estado da Bahia apresenta a maior prevalência de acidentes escorpiônicos no Brasil (BRASIL, 2021).

\section{ASPECTOS ECOLÓgICOS E IMPACTOS CLIMÁticos DOS ACIDENTES POR ESCORPIÕES}

Estações climáticas de calor facilitam a reprodução de aracnídeos. Climas quentes e úmidos são perfeitos para o aparecimento dos escorpiões que podem ser encontrados devido a condições climáticas favoráveis, em todos os continentes, exceto na Antártida. Nas Américas, podemos encontrar escorpiões habitando desde regiões do Canadá até a Patagônia (LOURENÇO, 2002a).

Os escorpiões vivem tanto em desertos quanto em matas, em relação aos ecossistemas podemos encontrá-los em vários, exceto tundra, taiga de alta latitude, áreas boreais e regiões de elevada altitude (POLIS, 1990). Segundo pesquisas, já observaram escorpiões nos Andes peruanos com mais de $5.000 \mathrm{~m}$ de altitude, em interior de cavernas, embaixo das pedras cobertas de neve, florestas úmidas e desertos (BRASIL, 2001). No clima do Cerrado, o escorpião amarelo (Tityus serrulatus) é o mais prevalente devido a sua alta adaptabilidade as condições urbanas. São comuns nos estados de São Paulo e Minas Gerais.

Mesmos encontrados em regiões extremas os escorpiões são exigentes e previsíveis em relação aos habitats, observadas suas características climáticas biogeográficas e ecológicas. Espécies do gênero Centruroides, Isometrus, Tityus, Euscorpius e Bothriurus, são encontradas em ambientes alterados pela ação do homem (LOURENÇO; EICKSTEDT, 2009). 


\section{PATOGÊNESE}

A peçonha dos escorpiões é constituída por uma complexa mistura de proteínas de baixo peso molecular e uma pequena quantidade de aminoácidos, sendo solúvel em água. É produzida por um par de glândulas localizadas no telson, na extremidade da cauda do animal. Após ser inoculada pelo ferrão, a peçonha ganha a corrente sanguínea (CUPO et al.,2003; SIQUEIRABATISTA et al., 2020).

A inoculação da peçonha desencadeia dor local de intensidade variável e efeitos complexos nos canais de sódio. Estes efeitos produzem despolarização das terminações nervosas pósganglionares, ocorrendo liberação de adrenalina, noradrenalina e acetilcolina, além de cortisol, glucagon, insulina e angiotensina II (BRASIL, 2001; BRASIL, 2019).

Os mediadores liberados pela ação da peçonha são responsáveis pelas manifestações sistêmicas que podem ocorrer, sendo predominantes os efeitos simpáticos ou parassimpáticos, com atividade hemolítica, proteolítica, colinesterásica e fosfolipásica (BRASIL, 2001). Os acidentes escorpiônicos não se distinguem quanto ao mecanismo de ação da peçonha, podendo esta gerar manifestações clínicas semelhantes mesmo quando o acidente envolve espécies diferentes. Os acidentes envolvendo Tityus serrulatus são considerados mais graves visto que, nesses casos, são mais frequentes a ocorrência de manifestações sistêmicas (BRASIL, 2001; BRASIL, 2009; BRASIL, 2019).

As diferentes peçonhas escorpiônicas podem desencadear efeitos sistêmicos diversos, pois em sua composição contém neurotoxinas, cardiotoxinas, nefrotoxinas, toxinas hemolíticas histaminas, fosfolipases, fosfodiesterases, hialuronidases, triptofano e liberadores de citocinas que irão mediar o processo inflamatório (SILVA, 2013). Apesar de serem mais raros, os casos graves de envenenamentos por escorpiões decorrem dos efeitos das toxinas, destacando-se taquicardia, isquemia miocárdica consequente ao espasmo coronariano ou até miocardite, reações anafiláticas à peçonha devido aos componentes proteicos, sudorese, náuseas e vômitos, fenômenos trombogênicos, insuficiência renal aguda, hipotensão arterial, tremores, mioclonias, entre outros (SILVA, 2013; CAMPOS et al., 2020). 


\section{ASPECTOS CLÍNICOS}

O primeiro sintoma e, também, o mais comum após a picada de um escorpião é a dor local, que apresenta início imediato. Esta pode variar desde leve até intensa, sendo frequentemente necessária a realização de bloqueio da região da picada através da infiltração de anestésicos locais. A dor decorrente da picada pode irradiar para o membro afetado, podendo evoluir com parestesia da região, sudorese e piloereção (BRASIL, 2019).

Os acidentes podem ser classificados como leves - quando há apenas dor local associada ou não a parestesias -, moderados - quando além de dor local, ocorrem sinais e sintomas sistêmicos discretos, como sudorese, vômitos ocasionais, taquicardia, taquipneia e hipertensão arterial ou hipotensão arterial leves - e graves - quando o acidentado apresenta sudorese profusa, vômitos incoercíveis, salivação excessiva, queda importante do estado geral alternando com agitação psicomotora, bradicardia, insuficiência cardíaca e/ou renal, edema agudo de pulmão, choque, convulsões e coma (BRASIL, 2001).

Quando há excitação franca dos sistemas simpático e parassimpático, esta pode desencadear hiperfunção de glândulas nasais, sudoríparas - responsáveis pela sudorese característica de acidentes mais graves, pancreáticas e gástricas, o que desencadeia náuseas e episódios de vômitos, além de rinorreia e lacrimejamento. Pode ocorrer evolução para pancreatite e insuficiência renal aguda (ALBUQUERQUE et al., 2018; SIQUEIRA-BATISTA et al., 2020).

Manifestações cardíacas incluem arritmias, hipotensão - e, por vezes hipertensão - arterial, insuficiência cardíaca congestiva, edema agudo de pulmão, diminuição do débito cardíaco, podendo evoluir para choque. Ademais, são descritas a ocorrência de diminuição da temperatura corporal, miose, espasmos musculares e priapismo (SIQUEIRA-BATISTA et al., 2020). As manifestações neurológicas decorrentes do envenenamento por escorpiões variam desde sonolência ou agitação até tremores, hipertonia, confusão mental e coma (BRASIL, 2001).

A gravidade dos casos é dependente de distintos fatores, tais como espécie e tamanho do escorpião, quantidade de peçonha inoculada, idade e massa corporal do indivíduo acidentado, sensibilidade do acidentado à peçonha, diagnóstico precoce, tempo desde o acidente até a administração do soro antiescorpiônico e a manutenção de funções orgânicas vitais (BRASIL, 2001). Entre os diagnósticos diferenciais em acidentes escorpiônicos, pode se citar o botulismo, picadas de insetos, reações distônicas induzidas por medicação, difteria, coagulação intravascular 
disseminada, miastenia gravis, pancreatite, tétano, toxicidade por organofosforados e envenenamentos por Lonomia obliqua, Chilopoda, Micrurus lemniscatus, Atrax robustus, Latrodectus hasseltii, Latrodectus, Crotalus durissus, Theraphosidae (ALBUQUERQUE et al., 2018; SIQUEIRA-BATISTA et al., 2020).

\section{DIAGNÓSTICO}

O diagnóstico dos acidentes envolvendo escorpiões devem partir de uma suspeita clínica, a qual deve ser pautada pela realização de anamnese pormenorizada e exame clínico acurado. É importante salientar que, na vigência de acidentes com quaisquer animais peçonhentos, a captura do animal, quando possível, é indubitavelmente precípua para a realização de uma abordagem efetiva do caso, entretanto, isso nem sempre se sucede. Recomenda-se que se realize exames complementares para melhor estudo do caso (BRASIL, 2001; CHENG et al., 2014).

Os exames complementares incluem hemograma, eletrocardiograma (ECG), ecocardiograma e, em casos estritamente necessários, coagulograma, ionograma, fração MB da creatinofosfoquinase (CK-MB) e exames de imagem (SIQUEIRA-BATISTA et al., 2020). Ademais, sob manifestações sistêmicas graves, o indivíduo acidentado deve ser internado e submetido a uma investigação clínica mais detalhada para reconhecimento das reais disfunções de seu quadro (BRASIL, 2019).

Ao hemograma, achados como leucocitose com neutrofilia podem ocorrer. O ECG pode revelar arritmias, alterações isquêmicas ou mesmo alterações sugestivas de distúrbios hidroeletrolíticos. O ecocardiograma auxilia no estudo do bombeamento sanguíneo pelo coração. Outros exames como o coagulograma, ionograma, CK-MB e estudos de imagem, auxiliam no reconhecimento de complicações do envenenamento, como os distúrbios hidroeletrolíticos mencionados, fenômenos trombogênicos ou mesmo hemorrágicos e congestão pulmonar ou edema agudo de pulmão (BRASIL, 2001; SIQUEIRA-BATISTA et al., 2020). Nos casos graves, nos quais ocorrem manifestações sistêmicas importantes, pode-se lançar mão de outros exames investigativos para avaliação de diversas disfunções orgânicas, como troponina, exames preditivos de função renal, incluindo sumário de urina, função hepática, tomografia computadorizada, técnicas de imunodiagnóstico, entre outros (BRASIL, 2001; BRASIL, 2019; VELASCO et al., 2020). 


\section{TRATAMENTO}

O tratamento do escorpionismo se divide em três fases, sendo a primeira sintomática, a segunda específica e a terceira manutenção das funções vitais (BRASIL, 2019). O tratamento sintomático consiste no alívio da dor, através da infiltração de anestésico local - lidocaína a 2\% sem vasoconstritor no local da picada ou a administração de dipirona na dose de $10 \mathrm{mg} k \mathrm{~kg}$ de peso a cada seis horas. Além disso, nos casos refratários, pode-se optar pela utilização de analgésicos opioides. Os casos considerados leves podem ser observados por quatro a seis horas (BRASIL, 2001; VELASCO et al., 2020; BORGES et al., 2020).

O tratamento específico comumente deve ser estabelecido durante manifestações moderadas a graves dos acidentes e consiste na administração do soro antiescorpiônico (SAEsc) ou soro antiaracnídico (SAAr) aos pacientes acidentados, especialmente crianças. A administração do SAEsc ou SAAr deve ser realizada o mais rápido possível, por via endovenosa na dose preconizada de acordo com a gravidade do acidente.O soro é composto por anticorpos contra os antígenos da peçonha e atua neutralizando-a. Reações de hipersensibilidade ao soro são raras (BRASIL, 2001; BRASIL, 2019). A súmula do tratamento do escorpionismo é apresentada no Quadro 1.

Quadro 1. Número de ampolas de soro antiescorpiônico ou antiaracnídico específico de acordo com a gravidade do acidente.

\begin{tabular}{|c|c|c|c|}
\hline & Antivenenos & Gravidade & $\mathbf{N}^{\circ}$ de ampolas \\
\hline & & Leve: dor e parestesias locais & - \\
\hline & & $\begin{array}{l}\text { Moderado: dor local intensa associada a uma } \\
\text { ou mais manifestações (náuseas, vômitos, }\end{array}$ & 2 a 3 \\
\hline \multirow[b]{2}{*}{$\begin{array}{l}\text { Acidente } \\
\text { Escorpiônico }\end{array}$} & \multirow{2}{*}{$\begin{array}{c}\text { SAEsc }^{\mathrm{a}} \\
\text { ou } \\
\text { SAA }^{\mathrm{b}}\end{array}$} & $\begin{array}{l}\text { sudorese, sialorreia, agitação, taquipneia e } \\
\text { taquicardia) }\end{array}$ & \\
\hline & & $\begin{array}{l}\text { Grave: além das manifestações clínicas } \\
\text { citadas na forma moderada, há presença de } \\
\text { uma ou mais das seguintes manifestações: } \\
\text { vômitos profusos e incoercíveis, sudorese } \\
\text { profusa, sialorreia intensa, prostração, } \\
\text { convulsão, coma, bradicardia, insuficiência } \\
\text { cardíaca, edema pulmonar agudo e choque }\end{array}$ & 4 a 6 \\
\hline
\end{tabular}

aSAEsc = Soro antiescorpiônico.

bSAA = Soro antiaracnídico (Loxosceles, Phoneutria, Tityus).

${ }^{\mathrm{c} T e m p o ~ d e ~ o b s e r v a c ̧ a ̃ o ~ d a s ~ c r i a n c ̧ a s ~ p i c a d a s: ~} 6$ a 12 horas.

$1 \quad$ Adaptado de Cheng D, Bush SP, ScorpionEnvenomation. Disponível

em:<http://emedicine.medscape.com/article/168230-differential>

Fonte: Brasil (2019). 
A terceira fase do tratamento deve ser realizada em casos extremamente graves e consiste na manutenção dos sinais vitais. Nesses casos, o acidentado poderá apresentar disfunção de múltiplos órgãos e sistemas (DMOS) e necessitará de internação hospitalar e suporte intensivo. Além da administração da soroterapia específica em dose adequada, o acidentado deve ser abordado de acordo com as disfunções apresentadas (BRASIL, 2001).

Nesse contexto, na ocorrência de choque, poderão ser administradas drogas vasoativas e hidratação para manutenção da pressão arterial e perfusão adequada de órgãos e sistemas. $\mathrm{Na}$ vigência de agitação psicomotora intensa, pode-se lançar mão de benzodiazepínicos ou outros sedativos em doses adequadas. Para indivíduos com franca dispneia e que cursam com dessaturação de oxigênio, poderá ser ofertado oxigênio sob cateter nasal ou máscara. Outras abordagens terapêuticas deverão ser avaliadas individualmente de acordo com a necessidade do paciente (BRASIL, 2001; BRASIL, 2019; VELASCO et al., 2020).

\section{PROFILAXIA E CONTROLE}

É fato conhecido que os escorpiões habitam frequentemente espaços peridomiciliares, principalmente sob entulhos e acúmulos de matérias orgânicas. Diante disso, é plausível evitar o acúmulo desses materiais próximo a residências. De maneira análoga, é prudente examinar roupas e calçados, além de panos de chão e tapetes antes de usar estes objetos. Além disso, manter berços e camas afastados, pelo menos, 10 centímetros de paredes, bem como evitar que roupas de cama se encostem ao chão. Outro hábito peculiar no controle de escorpiões e outros insetos é a criação de aves, como a galinha doméstica, já que esta é predadora do animal. De resto, é importante salientar que os hábitos de prevenção contra acidentes escorpiônicos não devem se limitar apenas aos ambientes rurais, mas devem ser instituídos também em áreas urbanas (BRASIL, 2001; BRASIL, 2019; SIQUEIRA-BATISTA et al., 2020). 


\section{TÉCNICAS DE INTELIGÊNCIA ARTIFICIAL PARA O ESTUDO DO ESCORPIONISMO}

A Inteligência Artificial (IA) é um campo de estudo da Ciência da Computação responsável por projetar e construir máquinas para automatizar tarefas que normalmente requerem inteligência humana (SANTOS; VECHIO, 2020; SIQUEIRA-BATISTA; SILVA, 2019). As técnicas de IA têm chamado a atenção considerável da comunidade científica nos últimos anos, devido à sua capacidade de alcançar resultados promissores em diversos problemas de pesquisa (LOBO, 2017; BRAGA et al., 2019).

No que diz respeito aos estudos envolvendo escorpiões, a IA tem desempenhado um papel importante na bioinformática, na resolução de tarefas que, basicamente, vão desde o reconhecimento de espécies até a identificação de venenos. Cole e Brewer (2019), por sua vez, desenvolveram uma solução denominada TOXIFY, que se baseia em Redes Neurais Recorrentes para identificar uma infinidade de venenos de animais a partir de uma determinada sequência proteica. A partir de um estudo comparativo com o ToxClassifier (GACESA et al., 2016), os autores afirmaram que o TOXIFIY é a solução mais acurada e precisa para distinguir o veneno animal de proteínas não tóxicas.

Este tópico fornece uma miríade de oportunidades de pesquisa que são adequadas para a aplicação de técnicas de IA, como os seguintes caminhos notórios: (i) a previsão de áreas de pontos críticos de escorpiões por meio da análise de dados históricos, (ii) uma análise de ocorrências, a fim de prever o número de acidentes por um curto período no futuro, (iii) a aplicação de modelos de deep learning - tipo de aprendizagem de máquina que treina computadores para realizar tarefas como seres humanos, o que inclui de imagem e previsões (SOUZA et al., 2020) - para descobrir a espécie de um determinado escorpião pelo reconhecimento de imagem de suas feridas de mordida e (iv) o apoio de profissionais de saúde no diagnóstico e tratamento de pacientes acometidos. Trabalhos relevantes nas áreas citadas são altamente esperados, pois podem contribuir para a redução do número de acidentes e vítimas fatais. 


\section{CONSIDERAÇÕES FINAIS}

Diante da significativa importância médico-clínica do escorpionismo no Brasil, associada ao constante número de acidentes ocorridos, faz-se necessário o domínio acerca deste tema por parte dos profissionais da saúde. Atenção especial ao estudo do escorpionismo deve ser dada pelos médicos generalistas, uma vez que estes atuam nas unidades de Atenção Primária à Saúde e nos serviços de Pronto Atendimento em todo o país.

O presente estudo traz uma revisão narrativa sobre os diversos aspectos inerentes ao estudo e abordagem clínica do escorpionismo. Entretanto, trata-se de um tema que nunca deixa de ser um desafio para o médico assistente, uma vez que existem múltiplas espécies de escorpiões no território nacional.

Nesse sentido, o bom clínico deve estar preparado para contar com o advento de novas tecnologias, como a Inteligência Artificial, reconhecendo-se que esta já é uma realidade, e que pode ser crucial no reconhecimento da espécie envolvida no acidente e, assim, além de facilitar a abordagem adequada, promove a melhora da sobrevida dos pacientes.

\section{REFERÊNCIAS}

ALBUQUERQUE, P. L. M. M., MAGALHÃES, K. N., SALES, T. C., PAIVA, J. H. H. G. L., DAHER, E. F., SILVA JUNIOR, G. B. Acute kidney injury and pancreatitis due to scorpion sting: case report and literature review. Revista do Instituto de Medicina Tropical de São Paulo, 60, e30, 2018.

BORGES, A.; LOMONTE, B.; ANGULO, Y.; ACOSTA DE PATIÑO, H.; PASCALE, J. M; OTERO, R.; MIRANDA, R. J.; DE SOUSA, L.; GRAHAM, M. R. ; GÓMEZ, A.; PARDAL, P. P.O; ISHIKAWA, E.; BONILLA, F.; CASTILLO, A.; MACHADO DE AVILA, R. A. ; GÓMEZ, J. P. ; CARO-LÓPEZ, J. A. Venom diversity in the Neotropical scorpion genus Tityus: Implications for antivenom design emerging from molecular and immunochemical analyses across endemic areas of scorpionism, Acta Tropica, v. 24, p. 1-23, 2020.

BRAGA, A. V.; LINS, A. F.; SOARES, L. S.; FLEURY, L. G.; CARVALHO, J. C.; DO PRADO, R. S. Machine Learning: O uso da Inteligência Artificial na Medicina. Brazilian Journal of Development, v. 5, p. 16407-16413, 2019. 
BRASIL. MINISTÉRIO DA SAÚDE. Manual de diagnóstico e tratamento de acidentes por animais peçonhentos. $2^{\mathrm{a}}$ ed. Brasília: Ministério da Saúde, Fundação Nacional de Saúde, 2001.

BRASIL. MINISTÉRIO DA SAÚDE. Secretaria de Vigilância em Saúde. Departamento de Vigilância Epidemiológica. Manual de Controle de Escorpiões. $1^{\mathrm{a}}$ ed. Brasília: Ministério da Saúde, 2009.

BRASIL. MINISTÉRIO DA SAÚDE. Sistema de Informação de Agravos de Notificação (SINAN), 2021.Disponível em <https://portalsinan.saude.gov.br/>. Acesso em 03 de abril de 2021.

BRASIL. MINISTÉRIO DA SAÚDE. Secretaria de Vigilância em Saúde. Guia de Vigilância em Saúde. $3^{\text {a }}$ ed. Brasília: Ministério da Saúde, 2019.

BROWNELL, P.; POLIS, G. Scorpion biology and research. New York: Oxford University Press, 2001.

CAMPOS, L. L.; CARDOSO, F. L.; ANDRADE-FILHO, A. Fisiopatologia e tratamento dos efeitos cardiovasculares e pulmonares no envenenamento por escorpião. Revista Médica de Minas Gerais, v.30, p. 1-8, 2020.

CARMO, E. A; NERY, A. A.; PEREIRA, R; RIOS, M. A; CASOTTI, C. A. Factors associated with the severiry of scorpiopoisoning. Texto\&Contexto - Enfermagem, v. 28, n. 1, p. 1-14, 2019.

CHENG, D. Scorpion Envenomation: differential diagnoses. New England Journal of Medicine, v. 371, n. 16, p. 1557-1560, 2014.

CHIPPAUX, J.-P. Emerging options for the management of scorpion stings. Drug Design, Development and Therapy, v.6, p. 165-173, 2012.

CODDINGTON, J. A.; COLWELL, R. K. Arachnids: encyclopedy of biodiversity. San Diego: Academic Press City, 2001.

COLE, T. J.; BREWER, M. S. TOXIFY: a deep learning approach to classify animal venom proteins. PeerJ 2019; 7, e7200.

COSTA, G. G.; SEREJO, L. F. M.; COELHO, J. S.; CÂNDIDO, D. M.; GADELHA, M. A. C.; PARDAL, P. P. O. First report of scorpionism caused by Tityus serrulatus, described by Lutz and Mello, 1922 (Scorpiones, Buthidae), a species non-native to the state of Pará, Brazilian Amazon. Revista da Sociedade Brasileira Medicina Tropical, v. 53, p. e20190285, 2020.

CUPO, P.; AZEVEDO-MARQUES, M. M.; HERING, S. E. Acidentes por animais peçonhentos: escorpiões e aranhas. Simpósio: Urgências e emergências dermatológicas e toxicológicas. Ribeirão Preto, 2003. 
GACESA R.; BARLOW, D. J.; LONG, P. F. Machine learning can differentiate venom toxins from other proteins having non-toxic physiological functions. PeerJ Computer Science, v.2, p. e90, 2016.

LISBOA, N.S.; BOERE, V.; NEVES, F.M. Scorpionism in the far south of Bahia, Brazil, 20102017: case profile and factors associated with severity. Epidemiol. Serv. Saúde, v. 29, n. 2, e2019345, 2020.

LOBO, L. C.. Inteligência Artificial e Medicina. Revista Brasileira de Educação Médica, v. 41, n. 2, p. 185-193, 2017.

LOBO, L. C. Inteligência artificial, o Futuro da Medicina e a Educação Médica. Revista Brasileira de Educação Médica, v. 42, n. 3, p. 3-8, set. 2018.

LOURENÇO, W. R. Scorpiones. In: ADIS, J. Amazonian Arachnida and Myriapoda: identification keys to all classes, orders, families, some genera and lists of known terrestrial species. Moscow: Pensoft Publishes, 2002.

LOURENÇO, W. R. Scorpions of Brazil. Paris: Les Editions de 1'IF, 2002a. 320p.

LOURENÇO, W. R.; EICKSTEDT, V. R. Escorpiões de Importância Médica. In: CARDOSO, J. L. C. et al. Animais peçonhentos no Brasil: biologia, clínica e terapêutica dos acidentes. São Paulo: Sarvier, 2009.

LOURENÇO, W. R.; LEGUIN, E. A. The true identity of Scorpio (Atreus) obscurus Gervais, 1843 (Scorpiones, Buthidae). Euscorpius, n. 75, p.1-9, 2008.

POLIS, G. A.; SISSOM, W. D. Life history. In: POLIS, G. A.; SISSOM, W. D. The biology of scorpions. Stanford: Stanford University Press, 1990.

SANTANA, L. A.; VICARI, M. V.; MOTTA, O. J. R.; NOVELLI, M. M.; MONTENEGRO, S. S. P.; PEREIRA, S. O.; MACHADO, G. R.; SANT'ANA, J. L. P.; SILVA, E. Arachnidism in Brazil. Brazilian Journal of Health Review, v. 3, p. 5-15, 2020.

SANTOS, A. M. J; VECHIO, G. H. Inteligência Artificial, Definições e Aplicações. Revista Interface Tecnológica, v. 17, n. 1, p. 129-139, 2020.

SILVA, N. A. Estudo dos efeitos renais da peçonha do escorpião Tityus stigmurus (Thorrel, 1876) (Scorpiones: Buthidae) em perfusão de rim isolado de ratos. Dissertação (Mestrado em Biologia Animal) - Centro de Ciências Biológicas da UFPE. Universidade Federal de Pernambuco, 2013.

SIQUEIRA-BATISTA, R.; SILVA, E. Notas sobre os fundamentos matemáticos da Inteligência Artificial. Revista de Ciência, Tecnologia e Inovação, v. 4, p. 44-54, 2019. 
SIQUEIRA-BATISTA, R.; GOMES, A. P.; SANTOS, S. S.; SANTANA, L. A. Parasitologia: Fundamentos e Prática Clínica. 1 ${ }^{\mathrm{a}}$ ed. Rio de Janeiro: Guanabara Koogan, 2020.

TANIELE-SILVA, J.; MARTINS, L. G; SOUZA, M. B.; SOUZA, L. M; CARDOSO, R. M. B; VELASCO, S. R. U; RAMOS, G. D. S; MIRANDA, C. T; MOURA, A. A; ANDERSON, L.; BASSI, E. J. Retrospective clinical and epidemiological analysis of scorpionism at a referral hospital for the treatment of accidents by venomous animals in Alagoas State, Northeast Brazil, 2007-2017. Revista do Instituto de Medicina Tropical de São Paulo, v. 62, e26, 2020.

VELASCO, I. T; NETO, I. T; BRANDÃO, R. A; SOUZA, H. P.; MARINO, L. O.; MARCHINI, J. F. M; ALENCAR, J. C. G. Medicina de Emergência: Abordagem Prática. 14 ed. Barueri - SP: Manole, 2020.

\section{Declaração de Interesse}

Os autores declaram não haver nenhum conflito de interesse

\section{Financiamento}

Recursos próprios

\section{Agradecimentos}

Ao estimado Prof. Dr. Rodrigo SiqueiraBatista (FADIP, UFV e UFRJ) pelas sugestões apresentadas por ocasião da elaboração do texto.

\begin{abstract}
Colaboração entre autores
$O$ presente artigo foi escrito pelo Jader Lúcio Pinheiro Sant'Ana, Iram Borges de Moraes Rocha Filho e Gabriel Resende Machado em conjunto com os docentes Mathias Viana Vicari, Oswaldo Jesus Rodrigues da Motta, Eugênio da Silva e Luiz Alberto Santana. Todos os autores cuidaram da parte dissertativa do artigo e aprovaram a versão final do texto.
\end{abstract}

